gap $>\mathrm{g}:=$ SymmetricGroup $(4)$;

$\operatorname{Sym}\left(\left[\begin{array}{lll}1 & 4\end{array}\right]\right)$

15 : betti $(t$, Weights $\Rightarrow\{1$, gap $\}$ false

01234 gap> tblmod2:= CharacterTable( tbl, 2);

05 = total: 1413144 BrauerTable $(\operatorname{Sym}([1 \ldots 4]), 2)$

1: . 2242 gap> tblmod2 = CharacterTable $(t b l, 2)$;

Journal of Software for

01234 gap> libtbl:= CharacterTable( "M" );

$06=$ total: 1413144 CharacterTable( "M")

fail $\quad r i n g ~ r 1=32003,(x, y, z)$, ds;

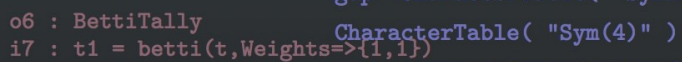

gap> CharacterTable( "Symmetric", 4 ) ; int a,b,c,t=11,5,3,0;

gap> ComputedBrauerTables ( tbl );

$\begin{array}{llrrr}0 & 1 & 2 & 3 & 4\end{array}$

[ , BrauerTable( Syu( [ 1 . 4 ]) poly $f=x^{\wedge} a+y^{\wedge} b+z^{\wedge}(3 * c)+x^{\wedge}(c+2) * y^{\wedge}(c-1)+x^{\wedge}$ $\mathrm{x}^{\sim}(\mathrm{c}-2) * \mathrm{y}^{\wedge} \mathrm{c} *\left(\mathrm{y}^{\sim} 2+\mathrm{t} * \mathrm{x}\right)^{\sim}-2$;

of = total: 1413144

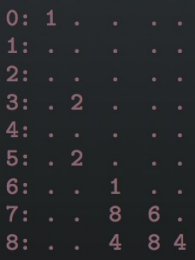

o7 : BettiTally

i8 : peek t1

$08=$ BettiTally $\{(0,\{0,0\}, 0) \Rightarrow 1\}$

$(1,\{2,2\}, 4) \Rightarrow 2$

$(1,\{3,3\}, 6) \Rightarrow 2$

(2, $\{3,7\}, 10) \Rightarrow 2$

$(2,\{4,4\}, 8) \Rightarrow 1$

(2, $\{4,5\}, 9) \Rightarrow 4$

$(2,\{5,4\}, 9) \Rightarrow 4$

(2, $\{7,3\}, 10) \Rightarrow 2$

$(3,\{4,7\}, 11) \Rightarrow 4$

$(3,45,5\}, 10$ Software for multiplier ideals

$(3,\{7,4\}, 11) \Rightarrow 4$

$(4,\{7,5\}, 12) \Rightarrow 2$

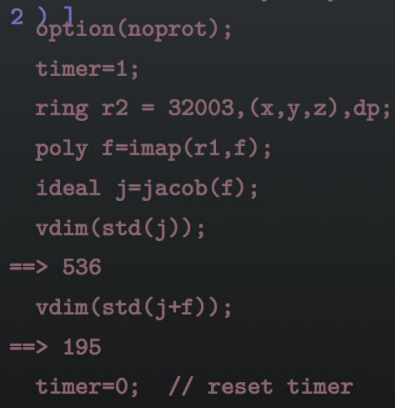




\title{
Software for multiplier ideals
}

\author{
ZACH TEITLER
}

\begin{abstract}
We describe a new software package for computing multiplier ideals in certain cases, including monomial ideals, monomial curves, generic determinantal ideals, and hyperplane arrangements. In these cases we take advantage of combinatorial formulas for multiplier ideals given by results of Howald, Thompson, and Johnson. The package uses the package Normaliz. It is available as a library for Macaulay2.
\end{abstract}

INTRODUCTION. Multiplier ideals have been applied to a number of problems in algebraic geometry in recent years, most spectacularly in recent major advances in the minimal model program [Hacon and McKernan 2007; Birkar et al. 2010] that built on earlier work showing the deformation invariance of plurigenera [Siu 1998]. Other applications include several results on singularities and linear series [Lazarsfeld 2004; Ein and Mustaţă 2006], a bound for symbolic powers [Ein et al. 2001], and applications to algebraic statistics [Watanabe 2009; Zwiernik 2011; Drton et al. 2009, Chapter 5]. New applications of multiplier ideals continue to emerge in topics such as Chow stability [Lee 2008] and singularities in generic liaison [Niu 2014]. With broad and growing interest in multiplier ideals, it is increasingly valuable to compute examples.

For a thorough introduction to multiplier ideals see [Lazarsfeld 2004]. Here is a definition of multiplier ideals in terms of resolution of singularities: Suppose $X$ is a smooth variety over a field $\mathbb{k}$ (we may assume $X$ is affine, or even just $\mathbb{k}^{n}$, since we are primarily interested in local issues), $I \subset O_{X}$ is a nonzero ideal sheaf, and $\mu: Y \rightarrow X$ is a $\log$ resolution of $I$, so that the total transform $I O_{Y}$ defines a divisor $F$ with simple normal crossings support, $F=\sum a_{i} E_{i}$, where the $E_{i}$ are distinct reduced components of $F$. Then for each real number $c \geq 0$ the $c$-th multiplier ideal is defined by

$$
\mathscr{g}\left(I^{c}\right)=\mu_{*} O_{Y}\left(K_{Y / X}-\lfloor c \cdot F\rfloor\right),
$$

where $K_{Y / X}$ is the relative canonical divisor of $Y$ over $X$, defined locally by the vanishing of the determinant of the Jacobian $d \mu$, and $\lfloor c \cdot F\rfloor$ denotes the componentwise round-down of the $\mathbb{R}$-divisor $c \cdot F$, given by $\lfloor c \cdot F\rfloor=\sum\left\lfloor c a_{i}\right\rfloor E_{i}$.

MSC2010: primary 14Q99, 14F18; secondary 13A15, 13P25.

Keywords: multiplier ideal, log canonical threshold, jumping number. 
In theory it is algorithmic to compute multiplier ideals by computing a resolution of singularities of $I$ followed by a sheaf pushforward. In practice it is more difficult; see [Frühbis-Krüger 2014].

Shibuta's [2011] algorithm for computing Bernstein-Sato polynomials and multiplier ideals via Gröbner basis methods in Weyl algebras (which he implemented in RisalAsir) was refined and implemented in the Dmodules library for Macaulay 2 by Berkesch and Leykin [2010]. The Dmodules library can compute multiplier ideals and jumping numbers of arbitrary ideals, but due to the difficulty of the computations can only handle modestly sized examples.

We describe a new software package named MultiplierIdeals that computes multiplier ideals of special ideals, including monomial ideals, ideals of monomial curves, generic determinantal ideals, and hyperplane arrangements, via combinatorial methods, using the Normaliz software and interface to Macaulay2 by Bruns, Ichim, and Kämpf [Bruns and Ichim 2010; Bruns and Kämpf 2010]. The combinatorial methods allow computations of somewhat larger examples than can be handled by general methods.

Wherever possible we work over an arbitrary field $\mathbb{k}$. Since multiplier ideals in our cases are computed by resolutions defined over $\mathbb{Z}$ (or over the $\mathbb{Z}$-algebra generated by the coefficients of the defining equations of the input data), we may work in arbitrary characteristic.

Our package also computes certain quantities associated to multiplier ideals: the log canonical thresholds and jumping numbers. Because of the round-down operation, $\mathscr{f}\left(I^{c+\epsilon}\right)=\mathscr{g}\left(I^{c}\right)$ for sufficiently small $\epsilon>0$. A real number $c \geq 0$ is a jumping number of $I$ if $\mathscr{g}\left(I^{c}\right) \neq \mathscr{F}\left(I^{c-\epsilon}\right)$ for all $\epsilon>0$. Every jumping number is in fact rational. The smallest strictly positive jumping number is called the log canonical threshold of $I$, denoted $\operatorname{lct}(I)$. It turns out that $\mathscr{g}\left(I^{0}\right)=(1)$ is the trivial ideal, so $\operatorname{lct}(I)$ is the supremum of $c$ such that $\mathscr{g}\left(I^{c}\right)=(1)$; equivalently, $\operatorname{lct}(I)$ is the first value of $c$ such that $\mathscr{E}\left(I^{c}\right) \neq(1)$.

The portion dealing with monomial ideals was written first and distributed as the package MonomialMultiplierIdeals. The portion dealing with monomial curves was written by C. Raicu, B. Snapp, and the author at the 2011 IMA Special Workshop on Macaulay2, and distributed as the package SpaceCurvesMultiplierIdeals. The portion dealing with hyperplane arrangements is based on code written by Denham and Smith for the HyperplaneArrangements package [2011]. These portions were all integrated into the present package, and computations with generic determinantal ideals added, at the 2012 Macaulay2 Workshop at Wake Forest.

Monomial ideals. For a monomial ideal $I \subset \mathbb{k}\left[x_{1}, \ldots, x_{n}\right]$, let monom $(I) \subset$ $\mathbb{Z}_{\geq 0}^{n}$ be the set of exponent vectors of monomials in $I$. The Newton polyhedron $\operatorname{Newt}(I)$ is the convex hull of monom $(I)$. Let $\mathbf{1}=(1, \ldots, 1) \in \mathbb{R}^{n}$. 
Multiplier ideals of monomial ideals are described by the following theorem of Howald:

Theorem 1 [Howald 2001; Blickle 2004]. The multiplier ideal $\mathscr{G}\left(I^{c}\right)$ is the monomial ideal containing $x^{v}$ if and only if $v+\mathbf{1} \in \operatorname{Int}(c \cdot \operatorname{Newt}(I))$. Here Int denotes the topological interior of $c \cdot \operatorname{Newt}(I)$ relative to the nonnegative orthant, that is, as a subset of $\left(\mathbb{R}_{\geq 0}\right)^{n}$.

In other words, the multiplier ideal is the quotient ideal

$$
\mathscr{g}\left(I^{c}\right)=\left(x^{v}: v \in \operatorname{Int}(c \cdot \operatorname{Newt}(I))\right): x^{\mathbf{1}} .
$$

The Newton polyhedron $\operatorname{Newt}(I)$ is defined by a system of inequalities $A v \geq b$, where $A$ is an $r \times n$ matrix, $b$ is a vector, and $\geq$ is the partial order of entry-wise comparison, where $a \geq b$ if and only if $a_{i} \geq b_{i}, 1 \leq i \leq r$. Then $c \cdot \operatorname{Newt}(I)$ is defined by $A v \geq c b$. The interior $\operatorname{Int}(c \cdot \operatorname{Newt}(I))$ is the solution of the system of inequalities given by

$$
\begin{cases}A_{i} v>c b_{i} & \text { if } b_{i} \neq 0 \\ A_{i} v \geq c b_{i}=0 & \text { if } b_{i}=0\end{cases}
$$

Since Newt $(I)$ is a rational polyhedron, we can (and do) take the $A$ and $b$ to have integer entries. Furthermore, since $I$ is an ideal the entries of $A$ and $b$ are nonnegative. In practice, it is sufficient to compute $\mathscr{E}\left(I^{c}\right)$ for rational $c=p / q$, and this can be done as follows: To find the integer vectors $v$ lying in the topological interior of the solution region to $A v \geq c b$ (equivalently, $q A v \geq p b$ ), we add 1 to the nonzero entries of $p b$, yielding a vector $b^{\prime}$ with entries $b_{i}^{\prime}=p b_{i}+1$ if $b_{i} \neq 0$, and $b_{i}^{\prime}=p b_{i}=b_{i}=0$ otherwise. Then the multiplier ideal $\mathscr{g}\left(I^{c}\right)$ is the quotient $\left(x^{v}: q A v \geq b^{\prime}\right): x^{1}$.

The software Normaliz can compute the defining inequalities $A v \geq b$ of $\operatorname{Newt}(I)$ and the solutions to the modified system $q A v \geq b^{\prime} ;$ Macaulay 2 can compute the ideal quotient by $x^{\mathbf{1}}$, giving the multiplier ideal:

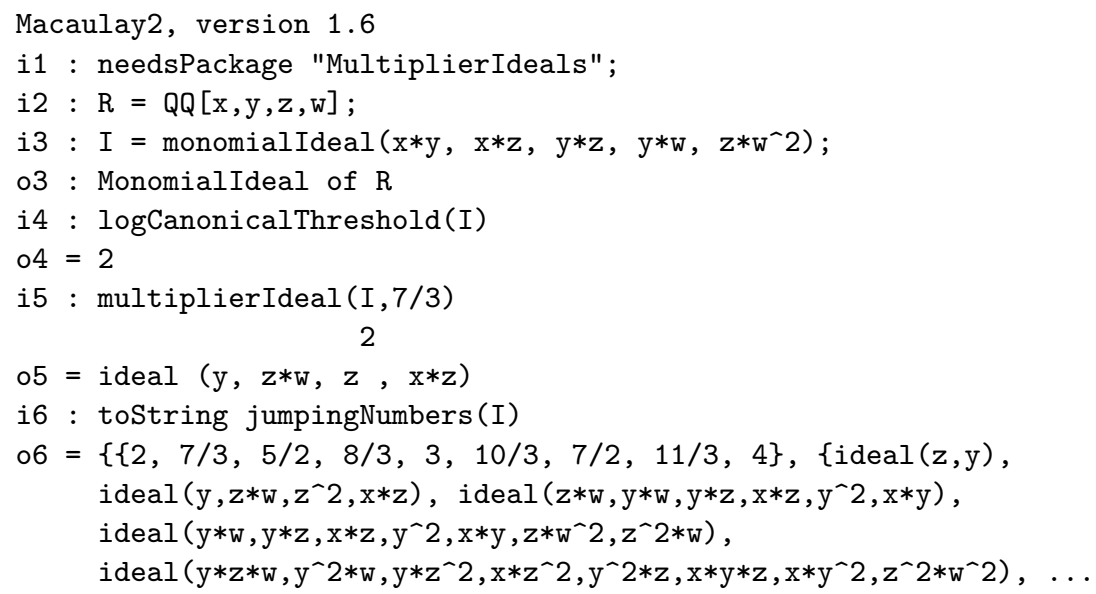


The jumpingNumbers command produces a list with two elements:

(1) A list of the jumping numbers of $I$ in the interval $(0, k(I)]$, where $k(I)$ is the analytic spread of $I$. A different interval may be specified as an optional argument.

(2) A list of the multiplier ideals at the jumping numbers. (The list is truncated in the above example.)

Thus the output of the last command says that this ideal $I$ has jumping numbers $2,7 / 3, \ldots$, and gives the corresponding multiplier ideals: $\mathscr{S}\left(I^{2}\right)=(z, y)$, $\mathscr{f}\left(I^{7 / 3}\right)=\left(y, z w, z^{2}, x z\right)$, and so on. Multiplier ideals and jumping numbers for $c>k(I)$ are given by Skoda's theorem [Lazarsfeld 2004, Theorem 9.6.21]. Namely, for $c>k(I), \mathscr{F}\left(I^{c}\right)=I \mathscr{F}\left(I^{c-1}\right)$; and $c>k(I)$ is a jumping number if and only if $c-1$ is a jumping number.

In the above example, the log canonical threshold, single multiplier ideal $\mathscr{f}\left(I^{7 / 3}\right)$, and list of nine jumping numbers and multiplier ideals were each computed in a fraction of a second on a 2012 MacBook with dual-core 64-bit $2.9 \mathrm{GHz}$ CPU and 8 GB RAM. By way of comparison, the Dmodules package takes about 42 seconds to compute the log canonical threshold on the same machine, and about 84 seconds to compute $\mathscr{f}\left(I^{7 / 3}\right)$. This comparison is only intended to illustrate the advantages of using special algorithms where available, and we remind the reader that the DModules package uses a general method.

For monomial ideals, extra information is available: for any monomial $x^{v}$, the package computes the threshold value $\min \left\{c: x^{v} \notin \mathscr{J}\left(I^{c}\right)\right\}$, and the list of facets of the Newton polyhedron that impose the nonmembership:

i7 : toString logCanonicalThreshold ( $\left.\mathrm{I}, \mathrm{z}^{\wedge} 2 \mathrm{*W}_{\mathrm{w}}\right)$

$07=(3$, matrix $\{\{2,2,1,1,-3\},\{2,2,0,1,-2\}\})$

This output means that $z^{2} w \notin \mathscr{g}\left(I^{3}\right)$ but $z^{2} w \in \mathscr{F}\left(I^{c}\right)$ for $c<3$. That is, for the exponent vector $v=(0,0,2,1), v+1$ lies on the boundary of $3 \cdot \operatorname{Newt}(I)$; and furthermore it lies on the intersection of two facets, the ones scaled up from the facets of $\operatorname{Newt}(I)$ defined by $2 x+2 y+z+w=3$ and $2 x+2 y+w=2$.

The $\log$ canonical threshold of the ideal $I$ itself is the threshold value for $1=x^{0}$.

Monomial CURVES. An affine monomial curve is one parametrized by $t \mapsto$ $\left(t^{a_{1}}, \ldots, t^{a_{n}}\right)$. We can and do assume that $1 \leq a_{1} \leq \cdots \leq a_{n}$ and $\operatorname{gcd}\left(a_{1}, \ldots, a_{n}\right)=1$. For convenience we denote this curve by $C\left(a_{1}, \ldots, a_{n}\right)$. It has a singularity at the origin when $a_{1} \geq 2$. The defining ideal is the kernel of the map $\mathbb{k}\left[x_{1}, \ldots, x_{n}\right] \rightarrow \mathbb{k}[t]$ given by $x_{i} \mapsto t^{a_{i}}$. This is a binomial ideal.

The multiplier ideals of affine monomial curves in dimension $n=3$ have been found by Howard Thompson [2014], ${ }^{1}$ using the combinatorial description of the

${ }^{1}$ This paper states the result over $\mathbb{C}$, but it holds over any field. 
resolution of singularities of a binomial ideal given in [González Pérez and Teissier 2002]. This yields a combinatorial formula in terms of the vector $(a, b, c)$ of exponents appearing in the parametrization $t \mapsto\left(t^{a}, t^{b}, t^{c}\right)$. Our software package implements Thompson's result, again calling on Normaliz to find generators for the semigroup of integer solutions to certain linear inequalities:

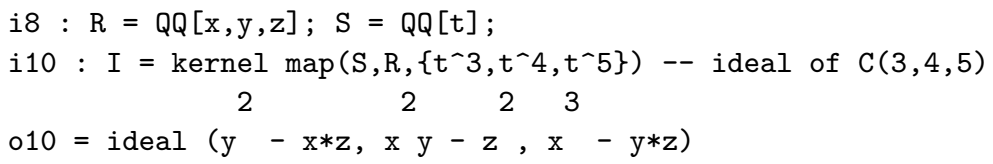

To compute the multiplier ideals and log canonical threshold of $I$, we input the list of exponents in the parametrization:

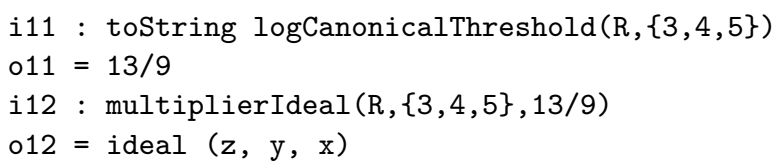

GENERIC DETERMINANTAL IDEALS. Let $X=\left(x_{i, j}\right)_{1 \leq i \leq m, 1 \leq j \leq n}$ be an $m \times n$ generic matrix, meaning one whose entries are independent variables. Let $I_{r}(X)$ be the ideal generated by the $r \times r$ minors of $X$. The multiplier ideals of $I_{r}(X)$ have been found by Amanda Johnson [2003]: ${ }^{2}$

Theorem 2. With $X, m, n$, and $r$ as above, the multiplier ideals are given by the following intersection of symbolic powers of determinantal ideals:

$$
\mathscr{S}\left(I_{r}(X)^{c}\right)=\bigcap_{i=1}^{r} I_{i}(X)^{(\lfloor c(r+1-i)\rfloor+1-(n-i+1)(m-i+1))} .
$$

Recall that symbolic powers of generic determinantal ideals may be expressed as

$$
I_{r}(X)^{(a)}=\sum_{\kappa_{1}+\cdots+\kappa_{s}=a} \prod_{i=1}^{s} I_{r-1+\kappa_{i}}(X),
$$

with the sum taken over partitions of $a$. See [Bruns and Vetter 1988, Theorem 10.4].

We may compute multiplier ideals of determinantal ideals in our software by giving the matrix $X$ and the size of minors. Here we examine multiplier ideals of the size- 2 and size- 3 minors of a $4 \times 5$ generic matrix:

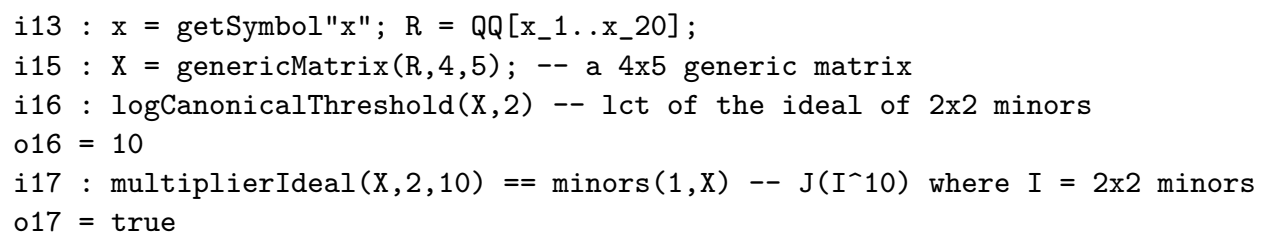

${ }^{2}$ This dissertation states the result for algebraically closed fields, but it holds over any field. 
i18: $\operatorname{multiplierIdeal}(\mathrm{X}, 2,11)==(\operatorname{minors}(1, \mathrm{X}))^{\wedge} 3--\mathrm{J}\left(\mathrm{I}^{\wedge} 11\right)$

$018=$ true

HYPERPLANE ARRANGEMENTS. A formula for multiplier ideals of hyperplane arrangements was found by Mustață [2006] and simplified in [Teitler 2008]. ${ }^{3}$ The HyperplaneArrangements package [Denham and Smith 2011] uses these results to compute multiplier ideals and log canonical thresholds of hyperplane arrangements. To this we add the ability to compute jumping numbers and other minor modifications. I thank Graham Denham and Gregory G. Smith, the authors of HyperplaneArrangements, for their permission to copy and modify their package's source code.

The following is Example 6.3 of [Berkesch and Leykin 2010]:

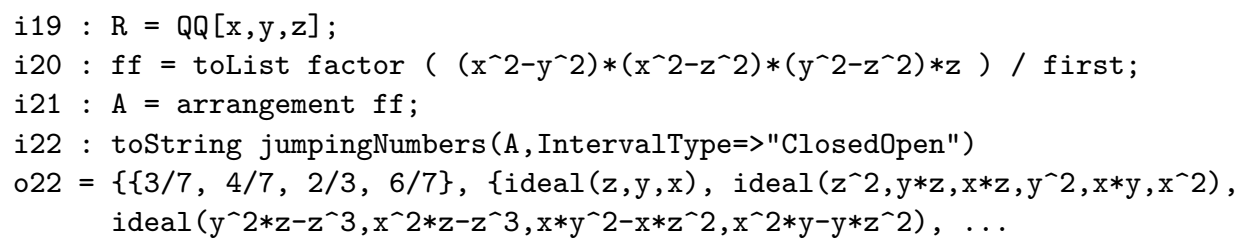

ACKNowledgements. I am very grateful to Claudiu Raicu and Bart Snapp for their critical contributions to the package and for a number of very helpful comments about this paper and the software package itself. I would also like to thank Howard Thompson for sharing his work-in-progress and for numerous helpful conversations, Graham Denham and Gregory G. Smith, the organizers of the 2011 IMA Special Workshop on Macaulay2, and the organizers of the 2012 Macaulay2 Workshop at Wake Forest.

\section{REFERENCES.}

[Berkesch and Leykin 2010] C. Berkesch and A. Leykin, "Algorithms for Bernstein-Sato polynomials and multiplier ideals", pp. 99-106 in Proceedings of the 2010 International Symposium on Symbolic and Algebraic Computation, edited by S. M. Watt, ACM, New York, 2010. MR 2920542

[Birkar et al. 2010] C. Birkar, P. Cascini, C. D. Hacon, and J. McKernan, "Existence of minimal models for varieties of log general type", J. Amer. Math. Soc. 23:2 (2010), 405-468. MR 2011f:14023 Zbl 1210.14019

[Blickle 2004] M. Blickle, "Multiplier ideals and modules on toric varieties", Math. Z. 248:1 (2004), 113-121. MR 2006a:14082 Zbl 1061.14055

[Bruns and Ichim 2010] W. Bruns and B. Ichim, "Normaliz: algorithms for affine monoids and rational cones”, J. Algebra 324:5 (2010), 1098-1113. MR 2011m:20001 Zbl 1203.13033

[Bruns and Kämpf 2010] W. Bruns and G. Kämpf, "A Macaulay2 interface for Normaliz", J. Softw. Algebra Geom. 2 (2010), 15-19. MR 2881130

[Bruns and Vetter 1988] W. Bruns and U. Vetter, Determinantal rings, Lecture Notes in Mathematics 1327, Springer, Berlin, 1988. MR 89i:13001 Zbl 0673.13006

\footnotetext{
${ }^{3}$ These papers state the result for $\mathbb{C}$, but it holds over any field.
} 
[Denham and Smith 2011] G. Denham and G. G. Smith, "HyperplaneArrangements, version 0.9 a package for Macaulay2”, 2011, http://www.math.uiuc.edu/Macaulay2/doc/Macaulay2-1.6/share/ doc/Macaulay2/HyperplaneArrangements/html/.

[Drton et al. 2009] M. Drton, B. Sturmfels, and S. Sullivant, Lectures on algebraic statistics, Oberwolfach Seminars 39, Birkhäuser, Basel, 2009. MR 2012d:62004 Zbl 1166.13001

[Ein and Mustață 2006] L. Ein and M. Mustață, "Invariants of singularities of pairs”, pp. 583-602 in International Congress of Mathematicians, II, edited by M. Sanz-Solé et al., Eur. Math. Soc., Zürich, 2006. MR 2007m:14050 Zbl 1096.14030

[Ein et al. 2001] L. Ein, R. Lazarsfeld, and K. E. Smith, "Uniform bounds and symbolic powers on smooth varieties”, Invent. Math. 144:2 (2001), 241-252. MR 2002b:13001 Zbl 1076.13501

[Frühbis-Krüger 2014] A. Frühbis-Krüger, "Desingularization in computational applications and experiments", pp. 269-284 in The resolution of singular algebraic varieties (Obergurgl, Austria, 2012.), edited by D. Ellwood et al., Amer. Math. Soc. and Clay Mathematics Institute, Providence, RI and Cambridge, MA, 2014. Zbl 1306.14001

[González Pérez and Teissier 2002] P. D. González Pérez and B. Teissier, "Embedded resolutions of non necessarily normal affine toric varieties", C. R. Math. Acad. Sci. Paris 334:5 (2002), 379-382. MR 2003b:14019 Zbl 1052.14062

[Hacon and McKernan 2007] C. D. Hacon and J. McKernan, "Extension theorems and the existence of flips", pp. 76-110 in Flips for 3-folds and 4-folds, edited by A. Corti, Oxford Lecture Ser. Math. Appl. 35, Oxford Univ. Press, 2007. MR 2359343 Zbl 1286.14026

[Howald 2001] J. A. Howald, "Multiplier ideals of monomial ideals", Trans. Amer. Math. Soc. 353:7 (2001), 2665-2671. MR 2002b:14061 Zbl 0979.13026

[Johnson 2003] A. A. Johnson, Multiplier ideals of determinantal ideals, Ph.D. thesis, University of Michigan, 2003, http://search.proquest.com/docview/287933180. MR 2704808

[Lazarsfeld 2004] R. Lazarsfeld, Positivity in algebraic geometry, II: Positivity for vector bundles, and multiplier ideals, Ergebnisse der Mathematik und ihrer Grenzgebiete (3) 49, Springer, Berlin, 2004. MR 2005k:14001b Zbl 1093.14500

[Lee 2008] Y. Lee, "Chow stability criterion in terms of log canonical threshold", J. Korean Math. Soc. 45:2 (2008), 467-477. MR 2009b:14091 Zbl 1144.14300

[Mustață 2006] M. Mustață, "Multiplier ideals of hyperplane arrangements”, Trans. Amer. Math. Soc. 358:11 (2006), 5015-5023. MR 2007d:14007 Zbl 1126.14003

[Niu 2014] W. Niu, "Singularities of generic linkage of algebraic varieties", Amer. J. Math. 136:6 (2014), 1665-1691. MR 3282984 Zbl 06380306

[Shibuta 2011] T. Shibuta, "Algorithms for computing multiplier ideals", J. Pure Appl. Algebra 215:12 (2011), 2829-2842. MR 2012f:14037 Zbl 1233.14015

[Siu 1998] Y.-T. Siu, “Invariance of plurigenera”, Invent. Math. 134:3 (1998), 661-673. MR 99i:32035 Zbl 0955.32017

[Teitler 2008] Z. Teitler, "A note on Mustaţă's computation of multiplier ideals of hyperplane arrangements", Proc. Amer. Math. Soc. 136:5 (2008), 1575-1579. MR 2008k:14005 Zbl 1157.14001

[Thompson 2014] H. M. Thompson, "Multiplier ideals of monomial space curves", Proc. Amer. Math. Soc. Ser. B 1 (2014), 33-41. MR 3168880

[Watanabe 2009] S. Watanabe, Algebraic geometry and statistical learning theory, Cambridge Monographs on Applied and Computational Mathematics 25, Cambridge University Press, New York, 2009. MR 2011g:62185 Zbl 1180.93108 
[Zwiernik 2011] P. Zwiernik, "An asymptotic behaviour of the marginal likelihood for general Markov models”, J. Mach. Learn. Res. 12 (2011), 3283-3310. MR 2012m:62248 Zbl 1280.68225

Received: 19 Feb 2014 Revised: 24 Apr 2015 ACCEPted: 5 Jun 2015

ZACH TEITLER:

zteitler@boisestate.edu

Department of Mathematics, Boise State University, 1910 University Drive, Boise, ID 83725-1555, United States 

Software for multiplier ideals

Zach Teitler

Partially ordered sets in Macaulay2

David Cook II, Sonja Mapes and Gwyneth Whieldon

Free resolutions and modules with a semisimple Lie group action

Federico Galetto

Computing characteristic classes and the topological Euler characteristic of complex projective schemes

Christine Jost 